\title{
»Une saison en enfer« Die erste Saison der Freien Bühne und Fontanes Kritiken
}

Die Liebe und der Suff, die regen den Menschen uff, pflegte meine Mutter bei Liebes- und Alkoholskandalen lakonisch zu kommentieren. Hätte sie die erste Saison der Freien Bühne in Berlin miterlebt, sie hätte reichlich Gelegenheit gehabt, diese menschliche Grundeinsicht zu zitieren: Ehebrüche, Verführungen, gescheiterte Lieben und Ehen und vor allem Alkoholiker, eine in der Dramenliteratur neue Erscheinung, wurden dem Publikum reichlich dargeboten, dazu Morde, Selbstmorde und eine beängstigende Zahl zerrütteter Familien. Anders als im klassischen Theater, das die menschlichen Katastrophen in historische oder mythische Ferne zu versetzen pflegt, um die conditio humana in der Ausnahmewelt der Fürsten und Helden $\mathrm{zu}$ erproben, fand das Unappetitliche und Verwerfliche, das Schändliche und Elende in der Freien Bühne hautnah, im Alltäglichen der Gegenwart statt. Ein radikaler Realismus erschien zum ersten $\mathrm{Mal}$ auf einer Berliner Bühne. Ein Teil der Protagonisten und auch Kritiker subsumierten diese neue Bühnenkunst unter dem damals modischen Begriff des Naturalismus - mit welchem Recht, ist nicht unumstritten.

Für die Fontaneforschung ist diese »Saison in der Hölle« deshalb relevant, weil der 70jährige Theaterkritiker Fontane, der seine 20jährige Tätigkeit als Rezensent der Vossischen Zeitung für das königliche Schauspiel gerade zu beenden im Begriff war, für sie auch diese Aufführungen besprach. Welches Verständnis er der dort gezeigten zeitgenössischen Dramatik entgegenbrachte, wird immer wieder durch seine Ibsen- und Hauptmann-Kritik belegt. Das übrige Programm und Fontanes übrige Kritiken dagegen werden kaum zur Kenntnis genommen. ${ }^{1}$

Um den vorherrschenden einseitigen Eindruck zu ergänzen, lade ich Sie ein, diese von der Parteien Haß und Gunst verwirrte, in der Fontaneforschung vernachlässigte Saison, die zu den historischen Ereignissen der deutschen Theatergeschichte gehört, Matinée für Matinée nachzuerleben, um sich die zum größeren Teil heute unbekannten Stücke zu vergegenwärtigen und von Fontanes Rezensionen, die immerhin ein Textkorpus von etwa 40 Seiten darstellen, einen Gesamteindruck zu erhalten.

Die am 5. 4. 1889 gegründete Freie Bühne ${ }^{2}$ leitete »ihre Daseinsberechtigung « aus dem "gegenwärtigen Zustand des deutschen Dramas $\aleph^{3}$ ab. Ihre Absicht war es, »unabhängig vo[m] [...] bestehenden Theater eine Bühne zu begründen, welche frei ist von den Rücksichten auf Theatercensur und Gelderwerb. Es sollen [...] etwa zehn Aufführungen moderner Dramen von her- 
vorragendem Interesse stattfinden, welche den ständigen Bühnen ihrem Wesen nach schwerer zugänglich sind. Sowol in der Auswahl der dramatischen Werke, als auch in ihrer schauspielerischen Darstellung sollen die Ziele einer der Schablone und dem Virtuosentum abgewandten lebendigen Kunst angestrebt werden. ${ }^{4}$

Um die Zensurbestimmungen zu umgehen, nach denen alle öffentlich aufgeführten Theaterstücke zur Prüfung ihrer »sicherheits-, ordnungs-, sittenoder gewerbepolizeilichen « Unbedenklichkeit eingereicht werden mußten, ${ }^{5}$ war die Freie Bühne als privater Verein konstituiert. Sie setzte sich zusammen aus 10 ordentlichen Mitgliedern, die das Programm bestimmten und persönlich - auch finanziell - hafteten, und etwa 1000 wohl vorwiegend männlichen außerordentlichen Mitgliedern, deren Recht lediglich darin bestand, für einen Jahresbeitrag den Theatermatinéen beizuwohnen, die einmal im Monat sonntags um halb zwölf mittags stattfanden. Jedes Stück wurde also nur einmal gespielt. Anders als ihr Vorbild, das 1887 gegründete Pariser Théatre libre, war die Freie Bühne ein »auf Erwerb nicht berechnete[s] Unternehmen «, ${ }^{6}$ aber da die Mitgliedschaften etwa 25000 Mark einbrachten, stand sie finanziell gesichert da. Die Direktion übernahm das 33jährige Gründungsmitglied Otto Brahm.

Es handelte sich von September 1889 bis Juni 1890 insgesamt um 9 Matinéen mit 10 Stücken. Das Repertoire bestand aus 4 deutschen, 1 französischen, 1 österreichischen, 1 russischen und 3 norwegischen Stücken. Bedenkt man, daß ursprünglich auch ein Strindberg-Drama gezeigt werden sollte, ${ }^{7}$ dann demonstrierte es überzeugend die avantgardistische Rolle des skandinavischen Gegenwartsdramas. Das Programm war sensationell: 3 Uraufführungen deutscher Stücke, einige deutsche und im übrigen - bis auf eine Ausnahme - Berliner Erstauffuhrungen. Es war wegen seines internationalen Charakters, gegen das der Vorwurf der »Ausländerei-Wirtschaft« und »Schmachvolle[n] Auslandsbevorzugung ${ }^{8}$ erhoben wurde, kontrovers. Die Freie Bühne, die seit dem 29.1. 1890 in der Wochenschrift Freie Bühne für modernes Leben auch ihr eigenes Publikationsorgan hatte, in dem 2 der Stuk$\mathrm{ke}^{9}$ zuerst publiziert wurden, verteidigte aber ihre Auswahl: Sie nahm »den weltlitterarischen Standpunkt Goethe's « in Anspruch, mit dem man unabhängig von nationaler Zugehörigkeit »die großen Dichter der Zeit« gerufen, statt deutsche literarische »Leichen aufgeputzt « habe. »Fast nur im Ausland fanden wir die elementaren Kräfte, die auf das junge Dichten der Heimath so fruchtbar wirken ${ }^{10}$ konnten; das Programm sei zudem durch die »aus Naturnotwendigkeit $«{ }^{11}$ gemeinsamen Ziele der internationalen modernen Literatur gerechtfertigt. Und sie argumentierte, daß zu ihrer Zeit schließlich auch »Lessing und Herder [...] in einer Zeit neuen Werdens und Gestaltens auf den Ausländer William Shakespeare enthusiastisch hinwiesen ${ }^{12}$ 
Eröffnung der Saison: 29. 9. 1889: Henrik Ibsen, Gespenster, ein Familiendrama in drei Akten, von 1883: Die Witwe Alving hat zum Andenken ihres Mannes ein Asyl gegründet. Ihr Sohn Osvald, ein Maler in einer schweren künstlerischen Krise, ist nach langer Abwesenheit aus Paris zurückgekehrt und wird von ihr vergöttert. Aber die Gespenster der Vergangenheit stehen gegen die Hoffnungen der Menschen auf. Frau Alving hat einst unter dem unsittlichen Lebenswandel ihres Mannes so gelitten, daß sie zum Schaden ihres Rufs als Frau und Mutter ihren siebenjährigen Sohn weggegeben hat und weggelaufen ist. Zu ihrem Entsetzen muß sie nun erkennen, daß ihr Sohn erblich durch seinen Vater affiziert ist: er trinkt und stellt den Mädchen nach. Ohne sein Wissen ist seine künstlerische Unfruchtbarkeit Folge seiner biologischen Belastung. Als er die bei seiner Mutter lebende Regine Engstrand heiraten möchte, enthüllt seine Mutter, daß sie als uneheliche Tochter ihres Mannes seine Halbschwester ist. Ein Feuer zerstört das Asyl und damit symbolisch das bisher so sorgsam gehütete bürgerliche Renommée des toten Alving. Frau Alving verspricht ihrem Sohn, ihn zu töten, falls seine gefürchtete Geisteskrankheit, offenbar Folge einer väterlichen Syphilis, ausbrechen sollte. Es geschieht Augenblicke später, während die Sonne strahlend aufgeht. "In sprachlosem Entsetzen" sieht sie den Verblödeten an. -

Das Stück war 1887 in Berlin in einer privaten Matinée schon gezeigt worden, Fontane hatte es rezensiert und sich dabei trotz aller Bewunderung für Ibsens »Kunst und Technik« $\left(691^{13}\right)$ ablehnend mit dessen pessimistischem sozialen Biologismus auseinandergesetzt. Seine Grundeinstellung änderte sich nicht mehr: künstlerische Anerkennung bei thematischer Distanz zu Ibsens »menschheitsbeglückenden Problemen« (848), vor allem seinem »Eheblödsinn $\ll .{ }^{14}$ Auch in seiner erneuten Kritik verwahrte sich Fontane gegen lbsens »These von der Erbkrankheit« als »Heimsuchung der Sünden der Väter an ihren Kindern« (808). Nur die künstlerische Überzeugungskraft des Dramatikers gebe ihr bei der Aufführung den Anschein von Wahrheit. Obwohl es Ibsen noch an der notwendigen künstlerischen Schönheit fehle, plädierte der 70jährige voller Überzeugung für die moderne Dramatik. Die »großen Schöpfungen« der Klassik, so Fontanes mutiges Bekenntnis, hätten aufgehört, »die Menschheit, >die jetzt dran ist $<$, noch lebhaft zu interessieren [...]. Und in dieser Not sprang der Realismus ins Dasein, der das Kunstheil auf dem entgegengesetzten Wege suchte. Wenn es das Paradies nicht mehr sein konnte, so sollt' es dafür ein Garten des Lebens sein« (809). Fontane stellte sich also in seiner ersten Kritik ganz demonstrativ hinter die Intentionen der Freien Bühne.

Zweite Matinée: 20. 10. 1889: Uraufführung von Gerhart Hauptmanns Erstlingsdrama, Vor Sonnenaufgang: Der sozialreformerische Alfred Loth mit 
hohen persönlichen Idealen von seelischer und körperlicher Gesundheit gerät bei Studien über die Lage der Arbeiter in Schlesien in eine durch den Verkauf ihres Landbesitzes reich gewordene, moralisch korrupte und trunksüchtige Familie: der Vater verbringt sein Leben in der Kneipe, die verheiratete Tochter, der schon ein Kind gestorben ist und die gerade ihr zweites, vermutlich ebenfalls lebensuntüchtiges Kind bekommt, hat die Trunksucht ihres Vaters geerbt, ihr im Materialismus versunkener Mann stellt seiner Schwägerin nach, die vulgäre Mutter unterhält ein sexuelles Verhältnis mit ihrem Neffen, dem Bewerber um die unverheiratete Tochter Helene. Nur diese, in Herrnhuth erzogen, leidet unsagbar unter den Familienverhältnissen. Zwischen ihr und Loth entwickelt sich eine Liebe, die zur Ehe führen soll, aber als er durch den Arzt des Hauses von der, wie er glaubt, erblichen Degeneration ihrer Familie erfährt, verläßt er sie gnadenlos, worauf sie Selbstmord begeht. -

Das Stück rief einen der großen Skandale der Theatergeschichte hervor. Ein Teil des Publikums lachte, grölte und buhte, womit es die zustimmende junge Gefolgschaft der neuen Literatur auf den billigeren Plätzen zu Szenenbeifall provozierte. Das Publikum folgte Loths - gekürzten - sozialethischen Erörterungen mit Abneigung - offenbar ein Plädoyer gegen die Abstraktheit von Loths Reformtheorien und »das furchtbare Gesetz der Vererbung - diese[n] Popanz der Naturalisten $\ll,{ }^{15}$ wie es Karl Frenzel in seiner Rezension formulierte. Zu Beginn des zweiten Aktes, als Kahl am frühen Morgen in derangierter Kleidung aus dem Schlafzimmer seiner Tante schleicht, rief ein in Berlin schon als Gegner und Störer alles Avantgardistischen einschlägig bekannter Arzt und Journalist: "Sind wir denn hier in einem Bordell?« und schwang im 5. Akt bei der Geburtsszene hinter den Kulissen eine Geburtszange.

Nicht minder aufgeregt und kontrovers war die Aufführung selbst. Ein

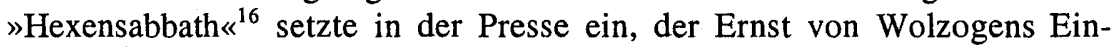
schätzung, »daß die heutige zweite Vorstellung [...] einen großen Mißerfolg des ganzen Unternehmens bedeutet $\ll,{ }^{17}$ schwer verständlich macht, denn sie verschaffte der Freien Bühne und Gerhart Hauptmann weiteste Publizität. Anhand des Dramas wurden moralische, politische und ästhetische Grundpositionen von traditionsverhaftet bis bedingungslos fortschrittlich erörtert. Es ging um kollidierende Wahrheits-, Wirklichkeits- und Schönheitsforderungen an die Gegenwartsliteratur, um das Recht der neuen Dramatik, ihren eigenen Gesetzen zu folgen. Vor Sonnenaufgang signalisierte, obwohl die Freie Bühne sich der »Notwendigkeit durchgreifender Aenderungen« gebeugt hatte, vielen Kritikern den Abstieg der Kunst in die politische Umsturzagitation, in die Gosse, die Schweinerei, die Vulgärsprache, in die bloße Afterkunst, ja das Ende der Kunst. Eine Ausnahme allerdings bildete die "Innigkeit, Reinheit und Wärme $\ll^{18}$ der »wunderbare[n] Liebesszene $\ll^{19}$ zwischen Loth und Helene im 4. Akt, die von der realistischen Drastik abstach und nahezu einhellig als 
bedeutend gewertet wurde. Die erste Kritik erschien - man höre und staune schon 4 Stunden nach dem Ende der Veranstaltung. Sie gab mit ihrer Verdammung der neuen Kunstrichtung den Ton an, den die meisten Kritiker nachsangen, kleidete sie aber in gekonnt formulierte Ironie:

Die Freie Bühne bewährt sich immer mehr als eine überaus sinnreiche Einrichtung, dem überfeinerten Großstädter die Sonntage angenehm durchzuekeln. Nachdem Ibsen, [...], mit etwas erblicher Gehirnerweichung und Wahnsinn den schönen Anfang gemacht, folgen [...] die Zauberlehrlinge, [...]. Man hatte das Gefühl, als seien die Vorstände des Vereins der Freien Bühne zugleich Vorstandsmitglieder des >Vereins gegen den Mißbrauch geistiger Getränke< und hätten einfach [...] die Vorlagen für die beiden Vereine vertauscht. [...] Was wollen sie uns noch vorsetzen, $[\ldots]$ nachdem sie uns heute schon das Abstoßendste geboten haben, was je auf einer deutschen Bühne erschien, $[\ldots] ?^{20}$

Die vorhergehende Aufführung von Gespenster verleitete die Kritiker zum Vergleich mit Hauptmanns Stück, das dann entweder als »eine lustige Parodie der Ibsenschen Gespenster « erschien, - so Karl Frenzel, ${ }^{21}$ der bar jeden Verständnisses für das Neue an der Dramatik dieser Jahre, Handlung und dramatischen Konflikt vermißte - oder als »die Erfüllung Ibsens« (820) - so Fontane, der sich weit über die vom Stoff niedergedrückten meisten Kritiker erhob, als er in seiner Rezension Vor Sonnenaufgang trotz des Zugeständnisses von »Schwächen und Unvollkommenheiten, ja selbst [...] Ridikülismen« (819) mit Formulierungen wie »ein furchtbares Haus, ein Haus mit einem Gespenst in jedem Winkel « (818), »durch das öde Haus hin klingt die Kunde von dem blutig Geschehenen «, »viel von der Ballade« (819) und »tragischerAusgang" (821) geradezu den Rang einer antiken Tragödie neuen Stils zusprach: Trotz ihrer »naturalistischen Derbheiten « übe Hauptmanns Kunst »eine heiligende, rettende Macht« aus und »verklär[e] das Häßliche« (820).

Dritte Matinée: 17. 11. 1889: Edmond und Jules de Goncourt, Henriette Maréchal, Schauspiel in drei Akten, von 1865, deutsche Erstaufführung: Auf einem Maskenball verliebt sich Paul de Bréville in Frau Maréchal, die in einer unerfüllten Ehe mit einem reich gewordenen ehemaligen Handwerker lebt. Der Zufall - das Stück ist voll davon - führt Paul als Verwundeten ins Haus Maréchal, wo er in der Hausherrin seine Angebetete wiedererkennt und mit ihr ein Verhältnis beginnt. Ihr Mann schlägt Paul als Ehemann für die Tochter Henriette vor, die Paul zwar liebt, aber die Beziehung ihrer Mutter errät und das ehebrecherische Paar selbstlos vor dem Vater deckt. Ein nächtliches Rendevous der Liebenden in Frau Maréchals Zimmer wird von ihrem Mann gestört. Er erschießt, einen Dieb vermutend, aus Versehen seine Toch- 
ter, die noch sterbend ihre Mutter schützt, indem sie Paul als ihren eigenen Geliebten ausgibt. -

Das wegen der Darstellung eines Ehebruchs und seiner realistisch- natürlichen Sprache (»une langue littéraire parlée«) revolutionäre französische Drama, das bei seiner Uraufführung 1865 einen Eklat ausgelöst hatte, hinterließ keinen tiefen Eindruck, obwohl an einigen Stellen gezischt wurde und einige Gegner von Hauptmanns Stück demonstrativ Beifall spendeten. ${ }^{22}$ Langeweile herrschte vor; Brahm selbst nannte es »nach zwei lauten Erfolgen einen sanften, aber deutlichen Mißerfolg ${ }^{23}{ }^{23}$ Aber auch hier gab es, wie der Vergleich von Fontanes und Wolzogens Kritik deutlich macht, kontroverse Urteile. Fontane lobte Mauthners Übersetzung des "geist- und witzsprühenden Dialogs « (833) als kongenial; Wolzogen fand sie bemüht, aber schon im Original »die längeren Reden der Hauptpersonen [...] völlig in dem herkömmlichen, beredten Feuilletonstil gehalten, der von Frankreich aus auch unser jüdisch-deutsches Salonstück infiziert hat « (S. 125). ${ }^{24}$ Wolzogen tadelte »die wahrhaft rührende Unbehilflichkeit der Technik « (S. 125), Fontane staunte über das »ausgezeichnete Stück [...] Welche Technik!« (831) Wolzogen vermißte die Natürlichkeit und beklagte vor allem die "Monologe der grausamsten Art« (S. 126); Fontane sah die Zufälle - »etwas Zufall ist überall« - aufgehoben in "Schicksal, Tragik (831). Einig waren sich beide in ihrer Kritik an dem »Engelscharakter der Tochter « (832), dem »Engel mit Schlagsahne« (Wolzogen, S. 127). Fontane gestand dem Drama insgesamt größeren Wert zu, nur war es ihm für die Freie Bühne nicht herausfordernd genug:

die Freie Bühne [...] muß Neues wählen, >wage zu irren nerspruch werden und ihre Niederlagen müssen ihr gerade so viel gelten wie ihre Siege. Ja, unter Umständen mehr. Eine Luftschiffahrts-Versuchsstation hat mehr Unglücksfälle zu verzeichnen als eine Feinbäckerei mit Ladenmamsells. Wiewohl bei letzteren schon der erste Schritt zur Gefahr gegeben ist. (831)

Vierte Matinée: 15. 12. 1889, vermutlich die deutsche Erstaufführung von Björnstjerne Björnson, Ein Handschuh, Schauspiel in drei Aufzügen, von 1883: Svava Ries, die ein Kinderasyl geschaffen hat, will sich mit Alf Christensen verheiraten, erfährt aber, daß er schon sexuelle Beziehungen gehabt hat - eine Art männliches Erbübel, denn ihre Empörung enthüllt, daß sowohl die Ehe ihrer als auch die von Alfs Eltern auf dieselbe Konstellation gegründet ist. Als Skandal begreifen alle nur das Aufdecken der männlichen Seitensprünge. Vor der Hochzeit wirft Svava Alf »ihren Handschuh ins Gesicht und stürzt nach links ab« - eine deutlich symbolische Geste, da ihr seine lockeren Sitten vertuschender Vater in einer früheren Szene seinen Handschuh angezogen hat. So endet das Stück, das von herausfordernden Gesprächen über Ver- 
erbung und die traditionelle Auffassung von Rolle und Wesen der Geschlechter und über soziale Probleme durchzogen ist, mit einer Kriegserklärung der jungen, mutigen Frau an die Männer. -

Dies die nicht gespielte endgültige Fassung des Stücks. Gegeben wurde eine frühere, nach der Svava schon im 2. Akt Alf mit dem Handschuh ohrfeigt, so daß am Schluß eine Versöhnung angedeutet wird. Dadurch wird nach Brahm der »unkünstlerisch abrupte [...] Schlu $\ll^{25}$ mit der Ohrfeige vermieden; nach Fontane wird Svavas Überzeugungskraft zerstört, denn »Svavas aus dem Hochgefühl der Liebe geborene Sittenstrenge« wird so »zu bloßer Spielerei und Laune« (838) reduziert. Der Erfolg der Vorstellung war schwach, denn das viele Theoretisieren der Gestalten ließ Langeweile aufkommen. Bezeichnenderweise wurden die zynischen Szenen, in denen sich erst die beiden Ehefrauen und dann die beiden Ehemännner zu ihrer anrüchigen Ehemoral bekennen, beklatscht, nicht Svavas Moralpredigten, obwohl diese doch Björnsons sozialpädagogische Botschaft enthalten. Fontane fehlte bei der von Svava »vertretene[n] Mischung von Wissenschaftlichkeit und Leidenschaft" (839) das Quentchen Humor.

Fünfte Matinée: 26. 1. 1890, wahrscheinlich die deutsche Erstaufführung von Graf Leo Tolstoi, Die Macht der Finsternis, Drama in fünf Akten, von 1886: Der reiche Bauer Peter wird von seiner zweiten Frau Anisja, die ein Verhältnis mit dem Knecht Nikita hat, auf Anraten von dessen Mutter vergiftet, da dieser sie verlassen will. Nikita, nun im Besitz von Peters Vermögen und mit Anisja verheiratet, verjubelt das Geld mit seiner Geliebten. Als sie ein Kind von ihm bekommt, bringen er, seine Mutter und Anisja es um und verheiraten das Mädchen. Aber vor seinem väterlichen Brautsegen schreckt Nikita aus Schuldbewußtsein zurück. Er beichtet statt dessen in der Hoffnung auf Gottes Vergebung vor den Hochzeitsgästen seine Sünden und wird verhaftet. Sein halb seniler, aber aufrechter alter Vater - daß er, durchaus symbolisch, Kloakenreiniger ist, wurde in der deutschen Übersetzung vertuscht wußte schon lange: »Eine Sünde nämlich hängt sich an die andre, zieht sie nach sich, und tief tief, Nikischka, bist Du versunken in der Sünde.« -

Statt der ungeheuer drastischen, ausgedehnten Mordszenen im 4. Akt (»Du hast's ja wie 'nen Pfannkuchen zerdrückt! Das ganze Köpfchen hast Du ihm zermalmt.«), wurde eine vom Dichter autorisierte Fassung des »Höllengemälde $[s] \ll^{26}$ gespielt, bei der der Mord, hinter die Bühne verlegt, nur dem Gespräch zwischen dem ahnenden alten Diener und der ahnungslosen jüngeren Schwester der Kindesmutter zu entnehmen ist. Bei der Aufführung hielten sich bis zum Ende des 2. Aktes Zustimmung und Ablehnung die Waage. Dann erhob sich der größte Teil des Publikums, »machte [...] Front gegen die Lärmmacher ${ }^{27}$ und erzwang Ruhe. Beklatscht wurden vor allem die Angriffe von Nikitas naivem Vater auf das Bank- und Profitwesen des Kapitalismus. 
Der 5. Akt, in dem sich auch »der Widerwilligste und Voreingenommenste« »dem gewaltigen Eindruck >des Menschen, der Buße thut [...] nicht entziehen $\aleph^{28}$ konnte, erhielt stürmischen Beifall. Er riß das Stück heraus, denn obwohl die breite Darstellung des bedrückenden Bauernmilieus gekürzt war, wurden die ersten vier Akte nicht nur von Fontane als zu lang und daher langweilig empfunden. Trotzdem stellte Tolstois Drama für den Kritiker die Erfüllung des neuen Theaters dar: "Die moderne realistische Kunst hat nichts Besseres und trotzdem wir überall in Nacht blicken, nichts heilig Leuchtenderes aufzuweisen als dieses Stück.« (841)

Fontane erläuterte nicht recht, warum, aber wer ihn kennt, weiß es. Bei Tolstoi wird menschliches Verbrechen aufgefangen in einer freiwilligen $\mathrm{Bu}-$ Be, durch die die moralische Welt wieder ins Lot gerückt wird. Der radikale Realismus bleibt hier nicht im Elend stecken, sondern läßt in der ethischen Verantwortung des Protagonisten schönheitliche Züge der menschlichen Existenz aufleuchten. So hatte es Fontane selbst auch an dem Helden des Romans dargestellt, an dem er gerade arbeitete, an Lehnert Menz in Quitt.

Sechste Matinée: 2. 3. 1890: die deutsche Erstaufführung von Ludwig Anzengruber, Das vierte Gebot, Volksstück in vier Akten, von 1877: Das Stück kreist um 3 sozial unterschiedlich angesiedelte Familien. 1. Der Hausbesitzer Hutterer hindert seine Tochter Hedwig daran, ihren Klavierlehrer Frey zu heiraten. Ihre erzwungene Ehe mit dem reichen Schürzenjäger und Trunkenbold Stolzenthaler ist unerfüllt. Das Sterben ihres siechen Kindes begreift sie als Folge des Lebenswandels ihres Mannes. 2. Bei Drechslermeister Schalanter gibt der faule Vater seinen Beruf auf, die Mutter verkuppelt ihre Tochter Josephe, und der Sohn Martin ist als disziplinloser Soldat »ein Trinker, ein Raufbold«. 3. Das Gärtnerehepaar Schön ist stolz auf seinen Priester gewordenen Sohn, der aber in seiner moralischen Blindheit für Hedwigs Ehe verantwortlich ist. Als Hedwig heimlich mit Frey, der unterdessen Martins gehaßter Feldwebel ist, ein Treffen vereinbart, verrät Schalanter es an ihren empörten Mann. Am Treffpunkt erschießt Martin voller Wut über dessen Vorwürfe Frey. Im unproportioniert kurzen vierten Akt bereut der Priester seinen Rat an den Vater Hedwigs, die sich Josephe Schalanter verwandt fühlt: »Ob an einen oder an mehrere, wir sind ja doch zwei Verkaufte." -

Nur in einer von der österreichischen Zensur fürchterlich verstümmelten Fassung hatte das "Volksstück « Ende 1877 in Wien unter Wegfall jeden Titels uraufgeführt werden können. Die Freie Bühne verhalf ihm zu neuem Leben. Es war ihr unbestrittenster Erfolg, weil es das gesamte Publikum in seinem starken Beifall und auch die Kritik in ihrer Zustimmung vereinte. Fontane verstieg sich zu dem aus der Distanz kaum mehr nachvollziehbaren Bekenntnis - das Stück erscheint aus heutiger Sicht melodramatisch und plakativ, - über die zweite Hälfte: »ich kenne überhaupt nichts, auch das Größte 
miteingerechnet, was erschütternder auf mich gewirkt hätte « (845). Er betonte zum einen »die Gewalt sittlicher Wirkung«, die statt der Ablehnung »die dankbare Bewunderung aller weltlichen und geistlichen Staats- und Volkslenker an der mittleren Donau hätte wachrufen müssen « (843), und verteidigte zum anderen den Volkscharakter des Stuicke: »Die Vornehmheit hat ihre Tage gehabt; heute geht ein demokratischer Zug auch durch die Kunst.« (844)

Siebte Matinée: 7. 4. 1890, ausgerechnet Ostermontag, die Uraufführung von Arno Holz und Johannes Schlaf, Die Familie Selicke, Drama in drei Aufzügen: Die Zuschauer erleben die Weihnachtsnacht der Buchhaltersfamilie Selicke, die deren Elend enthüllt: der Vater kommt angetrunken und wie immer voller Haß auf seine Frau erst nach 2 Uhr morgens nach Hause; die Frau jammert nur über die kaputte Ehe und ihre Lage und wünscht sich den Tod; die erwachsene Tochter lehnt, weil sie die Familie nicht zu verlassen wagt, den Heiratsantrag des gerade Landpfarrer gewordenen Untermieters $a b$, der seinen Glauben verloren hat; die schwerkranke kleine Tochter stirbt im Lauf der Nacht; der Quacksalber, der sie nicht retten kann, ist eine sozial engagierte, aber verpfuschte Existenz; die beiden Söhne leben in ständiger Angst vor dem Vater. Der Tod des Kindes, der einen kathartischen Effekt haben könnte, bewirkt nichts in den Menschen. Als Wendt am Ende beim unentwegten Läuten der Kirchenglocken das Haus verläßt, bricht die sich opfernde Toni fast zusammen, aber der Pfarrer verspricht: "Ich komme wieder."

Das 2. revolutionäre Stück der Saison, Zustands-, nicht Handlungsdrama, das - trotz der erheblichen Streichungen - in quälender Minutiosität mit Sprachfetzen und z. T. in Dialekt jammernde Menschen von kleinbürgerlicher Durchschnittlichkeit in einer unveränderlichen Lage vorführte - ein Menschenbild, das jeder Idee vom freien Willen Hohn sprach, löste mit einer »betäubenden akustischen Rauferei, zwischen Zischen, Applaus und Bravogedröhn $\aleph^{29}$ den zweiten Skandal aus. Als die Autoren sich von der Bühne verneigten, riefen nach Landau »hundert ${ }^{30}{ }^{30}$ nach Brahm »drei bis vier ${ }^{31} \mathrm{Zu}$ schauer »raus «. Die ablehnende Presse wiederholte das schon auf Hauptmann gemünzte Vokabular des Ekels. Ungeteiltes Lob fand das Stück kaum; wo man es verteidigte, räumte man ihm als Experiment ein Bühnenrecht ein, ohne zu wünschen, daß dieses "genre ennuyant ${ }^{32}$ mit seinem deprimierenden Sich-im-Kreis-Drehen nun »zum täglichen Brot der Nation« (847) werde. Die Zukunft des Naturalismus sahen Gegner wie Karl Frenzel ${ }^{33}$ und Befürworter wie Fontane ${ }^{34}$ in einer Ausweitung seiner stofflichen Basis. Aber Fontane ging in seiner Anerkennung weiter als alle anderen Kritiker, denn er betonte, daß Die Familie Selicke von allen gezeigten Stücken »auf Kunstart, Richtung und Technik « die bisher geltenden dramatischen Maßstäbe, über deren »Grenzlinie « auch Hauptmann und Tolstoi nur »in diesem und jenem hinauszugehen« (845) gewagt hätten, am radikalsten in Frage stellte, und erkannte 
ihm nicht nur ein Bühnenrecht zu, sondern »die Zukunft« (847). Trotz den unleidlichen Wiederholungen und dem unbehaglichen Realismus sei den Autoren diejenige »rätselhafte Modelung « gelungen, die recht eigentlich Kunst schaffe: "Wenn ich das kleine Lieschen Selicke bei Nachbarsleuten im Hinterhaus hätte sterben sehen, so ist es mir zweifelhaft, ob ich geweint hätte, dem kleinen Lieschen, das gestern auf der Bühne starb, bin ich unter Tränen gefolgt. Kunst ist ein ganz besonderer Saft.« (847)

Darauf: die deutsche Erstaufführung von Alexander Kielland, Auf dem Heimwege, Charakterbild in einem Akt, von 1886: Die Heimkehr des Kaufmanns Harald Nordahl, der wegen Unterschlagung im Zuchthaus sitzt, wird von seiner Frau, ihren Kindern, dem Verlobten ihrer Tochter und dem Pastor erwartet. Dieser predigt den Kindern zum Unbehagen Frau Nordahls, die die lauen moralischen Maßstäbe der Gesellschaft beklagt, ihre Familie ernährt und auch die Schulden ihres Mannes zurückgezahlt hat, die Schwäche der vergebungsbedürftigen menschlichen Natur. Als ihr Mann angekündigt wird, erwartet man, daß sie ihn willkommen heißt, aber sie macht ihm bei der ersten Begegnung klar, daß er Verzeihung erst nach einem Läuterungsproze $\beta$ erwarten kann. So verläßt er sie noch einmal, um sich auf den wahren »Heimweg "zu begeben: »Wir sehen uns bald wieder."-

Das »Charakterbild « des norwegischen Autors gestattete wegen seiner Kürze die Entwicklung von Charakteren nicht und ließ daher den didaktischen Zug hervortreten. Es stieß bei Fontane auf starke Ablehnung. Er setzte das »ziemlich verrückte [...] Stück, unerquicklich von Anfang bis Ende« negativ gegen Die Familie Selicke ab, weil sich bei ihm die latenten Mängel der norwegischen Dramatik in Vergrößerung zeigten: Es verwechsle in seinem "grenzenlose[n] geistige[n] Hochmut « die Demonstration »menschheitsbeglückender Probleme« und »groß und charaktervoll sein sollende [...], aber eigentlich alberne [...] Tiraden« mit »Natur und Wahrheit«. »Alles Mumpitz« (848), sagte Fontane.

Achte Matinée: 4. 5. 1890: wahrscheinlich die Uraufführung von Arthur Fitger, Von Gottes Gnaden, Trauerspiel von 1884: Das Stück spielt »im Herbst 1792« in einem »Deutsche[n] Kleinstaat am linken Rheinufer «. Die französische Revolution ist ante portas. Die Hofgesellschaft muß mit der Niederlage der Koalitionstruppen bei Valmy fertig werden. Liebe und Ehe der menschlichen, aber ihr Gottesgnadentum strikt vertretenden Herzogin Anna Dorothea mit ihrem Kindheitsfreund, dem Forstwärter Wolfgang, führt in der kritischen Lage zu unerträglichen Spannungen im rebellischen Land und zwischen den Partnern. Obendrein ist Wolfgangs Bruder Rochus ein Opfer der aristokratischen Willkür ihres korrupten Onkels und Feldherrn. Erst als die Rebellen unter Führung ihres Mannes gesiegt haben, entsagt Anna abdankend ihrem Gottesgnadentum. Während er das Revolutionsheer führt und 
"wie ein brausender Frühlingssturm unter die Völker fahren" möchte, lebt sie nun in ihrer Forsthütte und läßt ihren Onkel und Nachfolger entfliehen, der sich als rechter Feigling und arg reduzierter Hamlet entpuppt: "Nur nicht, nicht sterben! Sterben! Tot sein! Im Grabe liegen! 0! 0! 0! « Als wegen ihrer Nachlässigkeit »Anklage [...] wider [sie] erhoben « wird, stößt sie ihrem Mann ein Messer in die Brust, fragt ihn »nach seiner Wunde fühlend", »Schmerzt es sehr? «, wirft sich über den Sterbenden, sagt, sich erhebend, »legt ihn und mich in eine Gruft « und »wirft sich wieder über den Leichnam." Fitgers laienhaftes, klischeereiches und konventionelles Stück, das wegen seiner politisch umstürzlerischen Botschaft von der Zensur verboten war, fiel gründlich durch. Bis auf die gespannte Aufmerksamkeit weckende 5. Szene des 4. Aktes, wo der Tagelöhner Bachler der Fürstin das Elend »von Bettlern, Krüppeln, Kranken, Hülflosen aller Art « vorhält, wurde die Vorstellung von Lachen, Höhnen und Grölen begleitet. Obwohl Fontane es als »Scherz《 abtat, daß die Freie Bühne »mit Hilfe dieses Stücks der >alten Richtung «, den Sieg der >neuen ‘ aufs eviden[te]ste hätte erweisen wollen « (849), beutete diese den Mißerfolg doch zu ihren Gunsten aus: "Man hat von uns verlangt, wir sollten nur vernachlässigte vaterländische Ware bringen«, aber wenn das »vielbegehrte Fitger'sche Drama ${ }^{35}$ durchfalle und auch die riskante moderne deutsche Dramatik auf Widerstand stoße, dann sei der hohe Prozentsatz an ausländischer Dramatik im Programm doch gerechtfertigt. Brahm feierte in seiner Kritik in der Freien Bühne den 4. 5. 1890 schlicht als »den Todestag der historischen Tragödie alten Stiles «, ${ }^{36}$ aber ihm wurde von Maximilian Harden widersprochen, der dem Stück eine gewisse Modernität zubilligte, es nur beklagte, daß die »skandinavischer« als Ibsens Nora angelegte Fürstin »ein blutleer einherschleppender Schemen ist, ein Experimentierweib aus einer dramatischen Klinik «. ${ }^{37}$ Nur künstlerische Blindheit konnte dem Stück bescheinigen, es stelle in Wolfgang »einen geistig ungewöhnlichen Mann« und in der Fürstin »eine Gestalt voll sittlicher Größe dar «. ${ }^{38}$ Fontane dagegen bezeichnete Wolfgang zurecht als »Blagueur comme-il-faut« und überhaupt den Fitgerschen Bastard aus Ibsen (»soziales Tendenzstück») und Schiller (»politisches Tendenzstück «, 849) als durch und durch unwahr: "Das Stück ist im wesentlichen veraltet und gehört vorwiegend jenen politischen Dramen aus vorfitgerscher Zeit an, wo ein Polizeiverbot das Glück und den Ruhm eines Stückes macht. [...] Für die Kunst ist es bedeutungslos« (851).

Neunte Matinée: 1. 6. 1890: die Uraufführung von Gerhart Hauptmanns 2. Stück, Das Friedensfest, Eine Familienkatastrophe in drei Vorgängen: Wieder wird ein Weihnachtsfest, Symbol familiärer Verbundenheit und der Erlösung von menschlicher Schuld, zur Folie eines familiären Zusammenbruchs. Das Leben von Dr. med. Scholz' Familie endete vor sechs Jahren mit dem Weggang des problematischen Vaters und der beiden Söhne, weil der jüngere 
den Vater geohrfeigt hatte. Zurückkehrend bringt der unterdessen 26jährige Wilhelm nun seine Verlobte und seine zukünftige Schwiegermutter ins Haus, die die Familie aussöhnen möchten. Er findet seine jammernde Mutter, seine gereizte altjüngferliche Schwester, seinen desillusionierten Bruder und seinen unerwartet heimgekehrten 68jährigen Vater. Beim brennenden Tannenbaum werden alle alten Feindschaften versöhnt. Aber Frau Scholz' Defaitismus, "Da mag man wollen und wollen [...], alles bleibt doch beim Alten «, der die eifrig betriebene Schuldsuche und -zuweisung sinnlos macht, bestätigt sich. Die latente Feindschaft der Brüder bricht wieder aus; der ältere verläßt das Haus; der geistesgestörte, schwerkranke Vater stirbt. Doch obwohl der Schatten des bedenklichen biologischen väterlichen Erbes über ihm hängt, bekennt sich Wilhelm zu seiner Verlobten und geht "Hand in Hand mit dem Mädchen aufrecht und gefaßt« von der Bühne. -

Mit Hauptmanns zweitem Drama endete die erste Saison der Freien Bühne zum einen mit einem Publikumserfolg, denn die Zuschauer folgten der Aufführung mit schweigender Aufmerksamkeit, und in den Beifall, besonders am Ende des 2. »Vorgangs«, mischte sich nur wenig Zischen; und zum anderen, da das Stück Fontane »ehrfurchtsvoll zugeeignet« war, mit einer Huldigung an ihn. Der Kritiker ließ sich davon nicht beeinflussen, sondern setzte Das Familienfest negativ von dem »genialer[en], kühner[en], unterhaltlicher[en], dramatischer[en] «Vor Sonnenaufgang ab und fragte sich in Verkennung der Intention des Stücks, »ob nicht, unter Anfügung einer dem 2. Akt zu gebenden kurzen Schlussszene, ein zweiaktiges Friedensfest einem dreiaktigen vorzuziehen gewesen wäre. Das Stück schlösse dann energischer ab« (853).

Der Wunsch entstand aus dem Bedürfnis, "Abwechselung" in die "Elendigkeit« zu bringen: »Luft, Licht und Freude fehlen; die Unken klagen in einem fort und verkünden schlecht Wetter und das Wasser unten ist schwarz und der Himmel oben ist grau. Die Tristheit in unserem jungen Realismus dauert zu lange.«

Dieser Vorbehalt allerdings bezog sich ausschließlich auf das Fehlen des »richtigen Maß[es]« (854). Das Recht, ja die Verpflichtung der jungen Dramatik, soziale Studien, Experimente auf die Bühne zu bringen, bestritt er keineswegs. Sein kurzes Resümé der ganzen Saison dankte der Freien Bühne, der die Zukunft gerechter sein werde, für ihren Mut und ermunterte das zaghafte und verständnislose Publikum, nicht »als Versicherungskommissarius « ins zeitgenössische Theater zu gehen, wo man bereit sein müsse, »ins pfadlose Meer hinauszusteuern und nach neuen Inseln zu suchen« (856). 
Welchen Eindruck hinterlassen die erste Saison der Freien Bühne und Fontanes Kritiken?

Das Programm war nicht Ausdruck eines geschlossenen naturalistischen Konzepts; es bestand vielmehr aus einer Folge moderner Stücke - bis auf Goncourts von 1865 und Anzengrubers von 1877 stammten sie alle aus den 80er Jahren -, für deren Gesamtheit der umfassendere Begriff >Realismus، wohl der angemessenere ist, sofern er einschließt, daß darin aus ihrer Lage und ihrem Miteinander entstehende, aber darum nicht lösbarere Probleme gegenwärtiger Alltagsmenschen milieu- und sprachgetreu und ohne das Eingreifen schicksalhafter Mächte auf die Bühne gebracht werden. Schley sieht ihr Gemeinsames in der »Auflehnung gegen schablonenhafte Moral und innerlich hohle Konvention «. ${ }^{31}$

Der Untertitel von Hauptmanns Das Friedensfest, "eine Familienkatastrophe«, statt »eine Familientragödie«, enthielt die demonstrative Distanzierung vom klassischen Theater und seinem Schuldbegriff, die Verlagerung ins Menschlich-Durchschnittliche, die das Programm insgesamt auszeichnete. Aber auch der erste Teil des Untertitels, »Familienkatastrophe « sprach für alle Stücke, denn thematisch stand die anklagende Musterung des illusorischen, verlogenen Charakters der viktorianischen Familienideologie, bis hin zur Darstellung von Familiengreuel, im Zentrum des Programms. Es wurde kein einziges Drama gespielt, in dem nicht zerbrochene, zerbrechende oder auf Unredlichkeit aufgebaute Familien vorgeführt wurden; kein Wunder, daß Menschlich-Erhebendes kaum stattfand. Insofern traf die erste Saison der Freien Bühne ins Herz der wilhelmischen Gesellschaft, und die entschiedene Ablehnung der meisten ihrer Kritiker entsprang wohl auch einem Selbstschutz. Die faktisch absolute Autorität des Vaters wurde etwa bei Ibsen, Hauptmann, Björnson, Holz/Schlaf und Kielland - schon eine Generation vor dem Expressionismus - als Popanz entlarvt. Die Väter waren ihren Familien kein Vorbild; sie ruinierten sie. Frauen und Kinder waren ihnen entglitten oder forderten sie offen heraus. Selbst wenn das Norahafte in Svava in der gespielten Fassung von Ein Handschuh zurückgebogen wurde ins frauliche Opfer zugunsten von Ehe und Gesellschaft, konnten sich gerade die Frauen im Publikum, sofern sie emanzipatorischen Gedanken nachhingen und am trüben Familienleben auf der Bühne nicht verzweifelten, doch ermutigt fühlen.

Die Spannweite der Stücke war groß; sie reichte von der Konventionalität von Fitgers historischem Drama über die Bürgerlichkeit Ibsens, Kiellands und der Goncourts bis zur Radikalität eines echten Naturalismus bei Hauptmann und Holz/Schlaf. Das neue Ideal der »absolute[n] Wahrheit in der Kunst $\aleph^{40}$ wurde deshalb auch in sehr unterschiedlichem Maße verwirklicht, und das wird weitgehend auch durch die Altersunterschiede der Autoren reflektiert. 
Den ältesten, Edmond de Goncourt, *1822, und den jüngsten, Holz, *1864, trennen immerhin über 40 Jahre, die von den übrigen ziemlich gleichmäßig ausgefüllt werden: Ibsen, *1828, Jules de Goncourt, *1830, Björnson, *1832, Anzengruber, *1839, Fitger, *1840, Kielland, *1849, Hauptmann, *1862, Schlaf, ${ }^{*} 1863$. Doch wies Brahm zu Recht darauf hin, daß die Autoren der neuen Literatur »nicht 55 , sondern 25 « seien »und die deutsche Literatur mithin eine Zukunft $\aleph^{41}$ habe, denn außer Fitger waren gerade die deutschen Beiträger die einzigen erst in den frühen 60er Jahren geborenen.

Die Freie Bühne erfüllte ihre selbstgesetzte Aufgabe, dem Theater eine ernsthafte soziale und künstlerische zeitgenössische Funktion zurückzuerobern, schon in der ersten Saison überzeugend. Sie katapultierte Berlin, das theatermäßig jahrzehntelang als hoffnungslose Provinz gegolten hatte, ins Zentrum moderner, riskanter Bühnenkunst, die von nun an im Repertoire der etablierten Bühnen erschien. Daß sie 10 ernsthafte Stücke hintereinander aufführte, ohne dem Publikum auch seine gewohnte seichte Komödienware anzubieten, bewies Mut.

Was Fontanes Kritiken angeht, so stammten sie keineswegs, wie er selbst ironisierend schrieb, „von einem auf dem Aussterbe-Etat stehenden alten Herrn $\ll ;{ }^{42}$ sie waren vielmehr der Höhepunkt seiner Kritikertätigkeit überhaupt, denn es handelt sich dabei um seine geschlossenste Gruppe von Theaterrezensionen überhaupt, um seine konsistent engagiertesten und - wenn man die beiden Nachzügler über Hauptmanns Einsame Menschen (Januar 1891) und Die Weber (September 1893) als gewissermaßen dazu gehörig betrachtet - um sein letztes kritisches Wort. Dieses überragte an Haltung, Sprache und Substanz alles zur Freien Bühne kritisch Vorgebrachte, stellte aber auch den äußersten Punkt von Fontanes Verständnis der neuen Literatur dar, denn die eigentlich ins 20. Jahrhundert führenden Werke der 90er Jahre, etwa von Arthur Schnitzler, den Brüdern Mann oder Rainer Maria Rilke, hat er nicht mehr zur Kenntnis genommen.

1. Anstatt, wie die meisten seiner Kollegen, das Sensationelle herauszustreichen und das Neue zu bekämpfen, ohne sich auf es einzulassen, behandelte Fontane es mit einer Selbstverständlichkeit, die sein Erscheinen auf der Bühne als etwas völlig Normales erkennen läßt. Er brachte dem ganzen Unternehmen der Freien Bühne und jeder ihrer Matinéen Wohlwollen entgegen. Auch der Radau bei einigen Vorstellungen wurde bewußt unterspielt oder als Teil einer lebendigen Theaterszene gelassen hingenomrnen:

Mit Strafbestimmungen und Verurteilungen, gleichviel ob sie aus dem bürgerlichen Gesetzbuch oder aus der Ästhetik genommen sind, gibt man dem in schönster Blüte stehenden Kampfe nur einen Zusatz von Gift und Galle, während sich die Dinge, wenn man alles ruhig laufen läßt, schließlich von selber machen. Wir sind beim Experimentieren und alles, 
was sich gegen unsere Experimentierkünste sagen läßt, ist ihre Kärglichkeit und Schüchternheit. Der Mitbewerb ist viel zu klein und beschränkt (830).

Ohne daß das eigentlich Kritische darunter litte, spricht aus seinen Kritiken großer Respekt vor jedem künstlerischen Versuch, jedem künstlerischen Mut, jeder künstlerischen Leistung. Fontanes Kritiken sind das Gegenteil von Beckmesserei und kritischer Hysterie.

2. Gerade wer Vorbehalte gegen die gelegentliche sprachliche Pedanterie und Umständlichkeit vor allem des mittleren Journalisten und Kritikers Fontane hat, wird diese wunderbar entspannten Rezensionen mit ungemischtem Vergnügen lesen. In der Fülle der aus dem Alltag genommenen Vergleiche, in dem lockeren, unprätentiösen Vokabular (Hauptmanns »Schnapskomödie« und Tolstois »Knackkomödie «, 845) und in dem Parlando des Ganzen ist jede Spur von »Aufgesteiftem « - ein Fontanesches Wort - getilgt.

3. Knudsens wiederholter Hinweis auf Fontanes "gesunde[s] Verhältnis zum Leben «, »seine[n] gesunde[n] Menschenverstand « ${ }^{43}$ unterschätzt dessen kritische Leistung. Seine Kritiken sind nicht emotionale, sondern sehr rationale Bekenntnisse zur Berechtigung des Wandels künstlerischer Prinzipien, Stoffe, Formen und Sprachmittel, die an ein waches Bewußtsein sozialen Wandels gebunden sind, wie es prägnant in dem zur Verteidigung von Anzengrubers Volksstück geschriebenen »Die Vornehmheit hat ihre Tage gehabt; heute geht ein demokratischer Zug durch die Kunst« (844) zum Ausdruck kommt. Ja, manchmal geht ihm die Freie Bühne nicht weit genug. So konstatiert er schlicht, daß $»$ Vater und Mutter< in der Wüstheit unsrer großen Städte längst ihres heilig patriarchalischen Charakters entkleidet sind und viel tausendfältig, auch in ihrer eigenen Familie, zu Trägern und Kultivatoren jeder Art von Niedertracht geworden sind« (841).

4. Aber Fontane besteht darauf, daß die neue Kunst nicht eo ipso gut ist, sondern sich wie alle Kunst künstlerischen Kriterien zu stellen habe. Und in dieser Hinsicht sprechen seine Kritiken immer wieder seine Grundüberzeugung aus, daß der echte Realismus nicht im menschlichen Elend ertrinken darf, sondern die Proportionen von Lachen und Weinen, Freude und Jammer, Recht und Unrecht wahren müsse. Er baut auf einen Realismus, der über Hauptmann und Holz/Schlaf hinauskommen würde. Hat ihm auf lange Sicht das rein akademische Interesse an den Stücken der Genannten und die anhaltende Popularität etwa von Effi Briest und Der Stechlin Recht gegeben? 


\section{Anmerkungen}

${ }^{1}$ Es ist bezeichnend, daß Rüdiger Knudsen (Der Theaterkritiker Theodor Fontane. Berlin 1942) in seinem 41 Seiten langen Kapitel über die Freie Bühne Ibsen und Hauptmann (S. 159 - 191) 38 Seiten und den übrigen 8 Stücken lediglich anderthalb widmet. Auch die letzte, von Peter Goldammer herausgegebene Auswahl von Fontanes Theaterkritiken (Theodor Fontane, Die Saison hat glänzend begonnen. Theaterkritiken. Berlin 1998) enthält neben Tolstois Die Macht der Finsternis wieder nur die beiden erwähnten Rezensionen.

${ }^{2}$ Das Unternehmen der Freien Bühne ist mehrfach kompetent dargestellt worden, vgl. etwa Gernot Schley, Die Theaterleitung der Freien Bühne. Diss. Berlin 1966; Helmut Schanze, »Theater-Politik-Literatur, Zur Gründungskonstellation einer >Freien Bühne zu Berlin 1889«, in: Literatur und Theater im Wilhelminischen Deutschland, hg. von Peter Bayerdörfer u. a. Tübingen 1978, S. 275-291.

${ }^{3}$ Paul Schlenther, »Der Freien Bühne erstes Kriegsjahr. Ein Gegner der Sache«, in: Freie Bühne, H. 21, S. 561.

${ }^{4}$ Ders., Wozu der Lärm? Genesis der Freien Bühne. Berlin 1889, S. 5.

${ }^{5} \S 7$ der Polizeiverordnung vom 10. 7. 1851.

${ }^{6}$ Otto Brahm, Der Naturalismus und das Theater, 1891. Zit. nach: Naturalismus. Manifeste und Dokumente zur deutschen Literatur 1880-1900, hg. von Manfred Brauneck und Christine Müller. Stuttgart 1987, S. 273.

${ }^{7}$ Vgl. ders., »Die Eröffnung der Freien Bühne«, in: Die Nation, 5. 10. 1889. Zit. nach: $O$. B., Kritische Schriften über Drama und Theater, hg. von Paul Schlenther. Berlin 1913, S. $251 \mathrm{f}$.

${ }^{8}$ M. G. Conrad, in: Die Gesellschaft, März 1890, S. 403 f.

${ }^{9}$ Hauptmanns Das Friedensfest, H. 1, S. 16-30; H. 2, S. 49-6 1, H. 3, S. 7888), Killands Auf dem Heimweg (H. 10, S. 305-312).

${ }^{10}$ Schlenther, wie Anm. 3, S. 565 f.

11" "Die Freie Bühne in Berlin«, in: Berliner Tage-Blatt, Nr. 527, 16. 10. 1909, Abendausgabe, und Nr. 530, 18. 10. 1909, Abendausgabe. Zit. nach: Naturalismus, wie Anm. 6, S. 313.

${ }^{12}$ Brahm, wie Anm. 7, S. 254.

${ }^{13}$ Fontanes Theaterkritiken werden im Text mit Seitenangabe nach der Hanser Fontane Ausgabe, Abteilung III, Bd. 2 zitiert.

${ }^{14}$ An Guido Weiß, 14. August 1889 (HB 2.709).

${ }^{15}$ Karl Frenzel, in: Nationalzeitung, Nr. 575, 21. 10. 1889, Abend-Ausgabe.

${ }^{16}$ Hauptmann an Brahm, 5. 11. 1889. Zit. nach: Otto Brahm - Gerhart Hauptmann, Briefwechsel 1889-1912, hg. von Peter Spengel. Tübingen 1985, S.98. 
${ }^{17} \gg$ Freie Bühne. Berlins Publikum und Presse über Hauptmanns Drama >Vor Sonnenaufgang «, in: Die Gesellschaft, Dez. 1889, S. 1737.

${ }^{18}$ Paul Lindau, in: Berliner Tageblatt und Handelszeitung, Nr. 534, 21. 10. 1889, Abend-Ausgabe.

${ }^{19}$ Wolzogen, wie Anm. 17, S. 1736.

${ }^{20}$ Isidor Landau, in: Berliner Börsen-Courier, Nr. 533, 20. 10. 1889, Abend-Ausgabe.

${ }^{21}$ Zit. nach Wolzogen, wie Anm. 17, S. 1738

${ }^{22}$ Vgl. Wolzogen, in: Die Gesellschaft, Jan. 1890, S. 127.

${ }^{23}$ In Die Nation, 23. 11. 1889.

${ }^{24}$ Die folgenden Wolzogen-Zitate, wie Anm. 22.

${ }^{25}$ Brahm, wie Anm. 7, S. 279.

${ }^{26}$ Maximilian Harden, in: Die Gegenwart, Nr. 5, 1. 2. 1890, S. 78.

27»Hans Olden, Tolstoi und sein Berliner Publikum«, in: Die Freie Bühne, H. 1, S. 15.

${ }^{28}$ Schlenther, in: Die Freie Bühne, H. 1, S. 13.

${ }^{29}$ Landau, in: Berliner Börsen-Courier, 9. 4. 1890.

${ }^{30} \mathrm{Ebd}$.

${ }^{31}$ Brahm, »Raus! , in: Die Freie Bühne, H. 11, S. 317.

${ }^{32}$ Schlenther, in: Die Freie Bühne, H. 10, S. 302.

${ }^{33} \mathrm{Vgl}$. Frenzel, Erinnerungen und Strömungen. Leipzig o. J., S. 327: »Die Zukunft des Naturalismus in der Kunst beruht auf der Erweiterung seines Stoffgebiets, nur wenn er aus der dumpfen und stickigen Atmosphäre, in der er sich bisher bewegt hat, in die Freiheit der Natur, in die Weite der Welt, von häßlichen und verkommenen zu edeln und schönen Gestalten, von der Roheit zur Bildung, von dem immer sinkenden zu dem immer aufwärts strebenden Menschen sich rettet, wird er eine neue Phase in der Entwickelung bilden.«

${ }^{34} \gg$ Denn allerdings scheint der moderne Realismus eine traurige Tendenz nach dem Traurigen hin zu haben und mit dieser Tendenz muß er brechen, wenn er sich seiner Widersacher erwehren, wenn er leben will.« (Fontane, wie Anm. 13, S. 846).

${ }^{35}$ wie Anm. 3.

${ }^{36}$ Brahm, wie Anm. 7, S. 291. Da sich Fitger, der durch den Artikel verbittert wurde, nicht um die Aufführung beworben hatte, sondern sein Stück von der Freien Bühne angefordert worden war, wurde Brahm seine Distanzierung als Opportunismus ausgelegt (vgl. Schley, wie Anm. 2, S. 76).

${ }^{37}$ In: Die Nation, Nr. 32, 10. 5. 1890, S. 479. Harden veröffentlichte seine Kritiken in der Nation unter dem Pseudonym M. Kent. Ob er wohl zu seinem Urteil, das Stuick sei obsolet, denn »das Gottesgnadentum hat sich längst mit dem Volke vermählt, den Ehevertrag nennt man die Verfassung und es zeigt sich, daß diese [...] Vernunftsheirat mit den Jahren für beide 
Theile immer zuträglicher und behaglicher wird « (ebd.), 8 Jahre später noch gestanden hätte, als unter anderem das penetrante Insistieren Kaiser Wilhelms II. auf dem Gottesgnadentum zu Spekulationen über einen Staatsstreich seinerseits führte und Harden wegen Majestätsbeleidigung 6 Wochen im Gefängnis saß?

${ }^{38}$ Anonym, in: Der Kunstwart. Rundschau für alle Gebiete des Schönen 2 (Okt. '88-Sept. '89), S. 6.

${ }^{39}$ Schley, wie Anm. 2, S. 129.

${ }^{40}$ Brahm, wie Anm. 31, S. 319.

${ }^{41}$ Ders., wie Anm. 14, S. 720.

${ }^{42}$ An Paul Ackermann, 8. September 1889 (HB 3.719).

${ }^{43}$ Knudsen, wie Anm. 1, S. 164, 170 und ähnlich öfter. 